\title{
Primary reconstruction for spinal infections
}

\author{
Donald D. Dietze, Jr., M.D., Richard G. Fessler, M.D., Ph.D., and R. Patrick Jacob, M.D. \\ Department of Neurological Surgery, University of Florida, Gainesville, Florida
}

Primary reconstruction using bone grafts and instrumentation for spinal infections remains controversial. Between 1991 and 1993, 27 infections of the spinal column were treated at the Department of Neurosurgery of the University of Florida. Of the 27 cases 20 (six cervical, eight thoracic, and six lumbar spine) required surgical debridement and spinal reconstruction to maximize eradication of the infection and maintenance of spinal alignment. All of the cervical and lumbar cases were caused by bacterial infections, and two of eight thoracic cases were caused by tuberculous infections. Spinal arthrodesis was performed in all cases: interbody grafts were used in 18 procedures and posterolateral onlay grafts in 14 . Interbody grafts were autologous in 10 cases (six rib and four iliac crest) and homoplastic in eight (six fibular and two humerus). All of the posterolateral onlay grafts were autologous (three rib and 11 iliac crest). Spinal instrumentation was used in 15 cases: four with Caspar plates and 11 with posterior segmental fixation (five hook/rod constructs and six screw/rod constructs). Seventeen of 20 patients achieved improved clinical status postoperatively and 18 of 20 showed radiographic evidence of bone fusion. Antibiotic drugs were administered parenterally for an average of 6 weeks followed by a 3-month course of oral antibiotic medications. Tuberculous infections were treated for 1 year with antibiotic therapy. The average follow-up period was 37 months from surgery and 31 months after completion of treatment with antibiotic drugs. The authors conclude that primary arthrodesis and instrumentation can be performed in acute spinal infections; however, successful management depends on aggressive debridement of infectious foci and prolonged treatment with parenteral antibiotic drugs.

\section{Key Words * spinal arthrodesis * spinal instrumentation * spinal osteomyelitis * spinal discitis * Pott's disease * spinal abscess}

Spinal osteomyelitis is found in 2 to $7 \%$ of all cases of osteomyelitis and in adults represents the most common site for hematogenously acquired osteomyelitis.[5,21,24,30,34] Management goals for spinal infections (discitis, osteomyelitis, epidural abscesses) are as follows: 1) preservation of neurological function; 2) prevention of sepsis; 3) permanent eradication of infection; and 4) spinal stabilization. Aggressive debridement of abscesses and parenterally administered antibiotic drugs maximize the prevention of sepsis and eradication of infection. Aggressive decompression of neural tissue, parenterally administered antibiotic drugs, and spinal stabilization maximize the preservation of neurological function.

Spinal stabilization frequently requires bone fusion. Indications for fusion are preoperative spinal deformity and iatrogenically induced instability following decompression. However, fusion remains a controversial treatment in cases of infection,[3] with several challenges to be considered: 1) Should 
vertebral reconstruction be performed primarily or delayed? 2) If arthrodesis is required, should the graft be vascularized, autologous, or homoplastic? 3) If instrumentation would augment spinal stabilization, should it be performed primarily or delayed? 4) If instrumentation is used, should it be removed after successful bone fusion?

To address these questions, we conducted a retrospective analysis of spinal infections treated with primary vertebral reconstruction including bone grafting and instrumentation.

\section{CLINICAL MATERIAL AND METHODS}

We conducted a nonrandomized retrospective analysis of spinal infections treated with primary vertebral reconstruction between January 1991 and January 1993 at the Department of Neurosurgery of the University of Florida. The total number of spinal infections treated in our department during this time interval was 27 cases in 25 patients: two pediatric ( $<16$ years of age) and 25 adult cases. Iatrogenically acquired spinal infections such as postoperative infections and postprocedural epidural punctures were excluded. Twenty-six of the 27 cases were treated with surgical decompression to preserve neurological function. One pediatric case was treated with bed rest and parenterally administered antibiotic drugs, resulting in resolution of back pain and eradication of the infection. Of the 26 cases treated surgically, 20 (all adults) required reconstruction for spinal instability. Spinal instability was defined as a preoperative spinal deformity caused by vertebral body collapse to $50 \%$ of vertebral height and/or spinal angulation of more than 20š. $[3,13,36]$ Spinal translation greater than $5 \mathrm{~mm}$ was considered a sign of instability; however, dynamic radiographs were not obtained because all patients had progressive clinical symptomatology (pain or neurological deficit). Spinal instability was also defined as iatrogenically induced disruption of two of three spinal columns during surgical decompression.[6] All cases were treated with parenterally administered antibiotic drugs (average 6-week course) followed by a 5-month course of oral antibiotic medications for a total antibiotic course of 4.5 months. The two tuberculous infections were treated with triple oral antibiotic drugs (isoniazid, rifampin, and pyrazinamide) for 1 year. Case summaries are presented in Table 1 and 1A. 
TABLE 1

SUMMARY OF 20 CASES OF SPINAL INFECTION IN PATIENTS UNDERGONG INSTRUMENTATION*

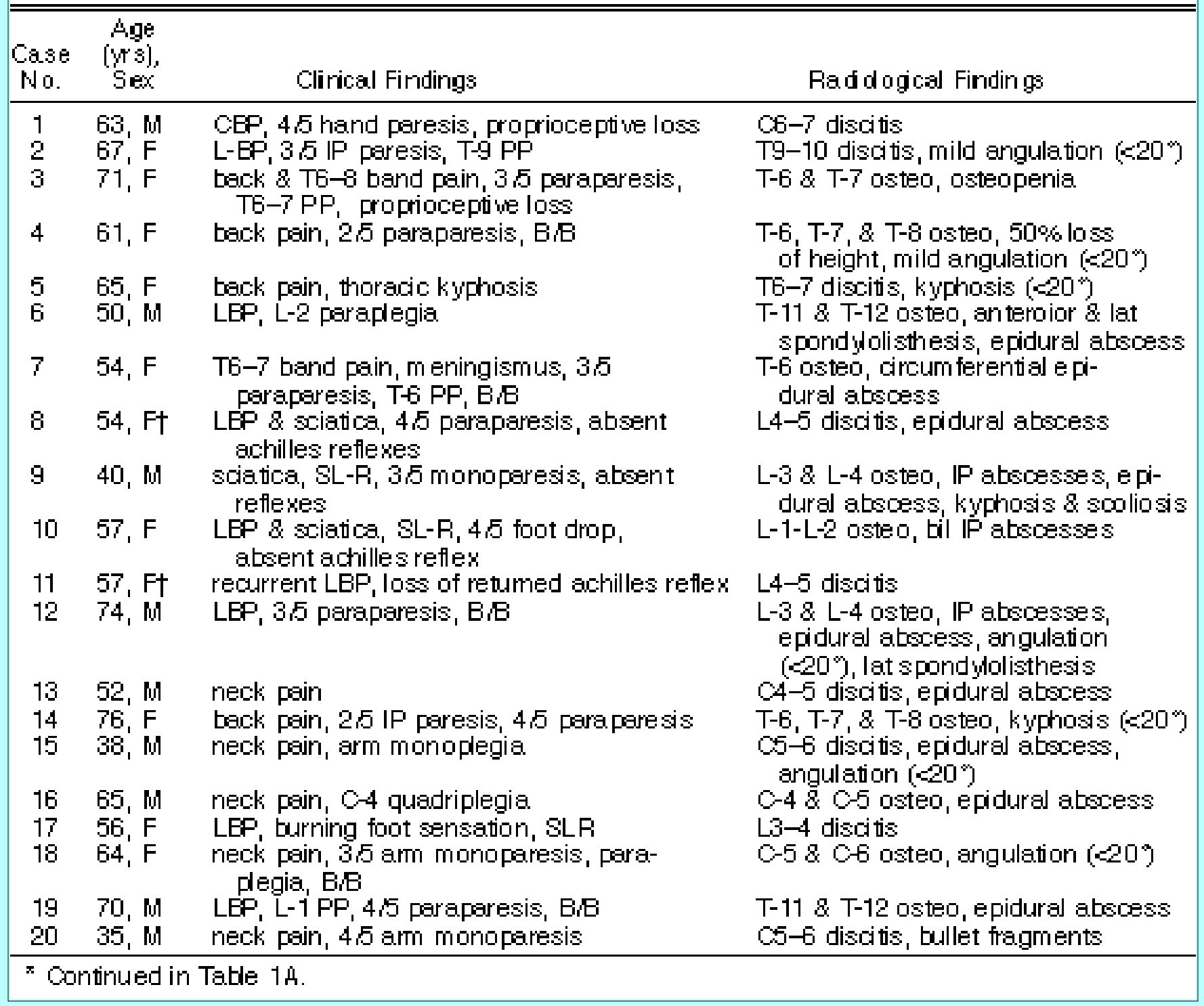


Case

No.
Strgical Treatment

C6-7 ACF, fibula allograft, halo orthosis

2 CTV, T9-10 discectom y' rib IB autograft, iliac crest OL autograft, damshell orthosis

3 LE, T-6, \& T-7 corpectomies, fibula IB allograft, ri b OL allograft, damshell orthosis

4 LEC, T-6, T-7, \& T-8 corpectomies, rib IB autograft, iliac crest OL autograft, T3-10 segmental fixation (hook hod), clamshell orthosis

$5 \quad \mathrm{EC}, \mathrm{T6}-7$ discectom $y$, rib IB autograft, iliac crest $\mathrm{OL}$ autograft, T3-10 segmental fixation (hook irod), clamshell orthosis

6 LE, T-11, a T-12 corpectomies, rib IB autograft, iliac crest OL autograft, T6-L2 segmental fixation (hook rod), clamshell orthosis

$7 \quad$ LE, T-6 corpectomy, vascularized rib IB autograft, iliac crest $\mathrm{OL}$ autograft, T-2-T-9 segmental fixation (hook rod), damshell orthosis

8 staged Th L4-5 discectom $y$, humerus IB allograft/ TP L-4 \& L-F decom pression, iliac crest OL autograft, L3-S1 segmental fixation (scremtrod), damshell orthosis

staged $L 2-5$ posterior decom pression, iliac crest $O L$ autograft , L2-5 segmental fixation (screwitod), RP L-3 \& L-4 corpectomies, iliac crest IB autograft, damshell orthosis

staged RP L-1 \& L-2 corpectomies, iliac crest IB \& OL autograft, T12-L3 segmental fixation (scremtod), damshell orthosis

11 staged RP L4-5 discectom y iliac crest İB autograft, drainage of IP abscessiliac crest OL autograft, L3-S1 segmental fixation (sorewhod), clamshell orthosis

staged RP L-3 \& L-4 corpectomies, humerus IB allograft, drainage of IP absoess L-3 \& $L-4$ laminectomies, iliac crest OL autograft, L2-5 segmental fixation (screwhod), clamshell orthosis

$\mathrm{ACF}, \mathrm{C}-4, \mathrm{a}$ C-5 corpectomies, fibula IB allograft, $\mathrm{C3}-6$ Caspar plate, hard collar

LC, T-6, T-7, \& T-8 corpectomies, vascularized rib IB autograft, rib OL autograft, damshell orthosis

$15 \mathrm{ACF}, \mathrm{C}, \mathrm{\&} \mathrm{C} 6$ corpectomies, iliac crest IB autograft, $\mathrm{C}-7$ Caspar plates, hard collar

$16 \mathrm{ACF}, 4-4, \&$ \& 5 corpectomies, fibula IB allograft, $C 3-6$ Caspar plate, hard collar

$17 \quad \mathrm{~L}-3$ \& L-4 laminectomies, L3-4 discectom y iliac crest $\mathrm{OL}$ auto graft, L2-5 segmental fi wation (hook trod), clamshell orthosis

ACF, C5, \& C6 corpectomies, fibula IB allograft, C4-7 Caspar plate, hard collar

19

TP T-11 \& T-12 decom pression, iliac crest OLautograft, T10-L1 segmental fixation (screwhrod), damshell orthosis

20
Organism

Found in

Culture

S. aureus

S. aureus

negative

(prior abx)

S. aureus

Salmonella

S. epidermidis

S. aureus

S. aureus

M. tuberculosis

S. aureus

S. aureus

S. aureus

S. aureus

M. tuberculosis

S. aureus

S. aureus

negative

Proteus

S. aureus

negative

(prior abx)
Risk F actors

IDDM, CRF

Pyelonephritis, IDDM, PVD, CA D, hypertension, obesity

Pyelonephritis, CAD, hypertension, obesity

NIDDM, hypertension, obesity

hypertension, T-6 compression fracture

pyelonephritis, SEE, CAD

ethanol abuse, smoker, obesity

ethanol abuse, smoker, obesity

PPD, IV drugs

Pyelonephritis, steroiddependent RA, smoker pyelonephritis, steroiddependent RA, smoker

IDDMI

epidural steroids

treast ca, spinal XRT, minor trauma.

minor trauma, lung ca, smoker ethanol abuse, UTI

hypertension

sepsis, Gl endoscopy

IDDM, CRF, PVD

GS'W' to neck

" $\mathrm{Abx}=$ antitiotic drugs; $\mathrm{ACF}=$ anterior cervical fusion; auto = autologous; $\mathrm{B} B \mathrm{~B}=\mathrm{bowel}$ and thadder dystunction; ca $=$ cancer; $\mathrm{CAD}=$ coronar yartery disease; $\mathrm{CEP}=$ cer vicotrachial pain; $\mathrm{CRF}=$ chronic renal failure; $\mathrm{CTV}=\cos$ totranswersectom y approach; GI = gastrointestinal; GSW' = gunshot wound; IB = interbody; IDDM = insulin-dependent diabetes mellitus; IP = iliopsoas muscle; IV = intrawenous; LEP = low-back pain; LE C = lateral extracavi tary approach; M. tuberculosis = Myoo bacterium tuberculosis; NIDDM = non-insulin dep. diabetes; $O L=$ onlay, osteo = osteom yelitis; PP = pinprick loss of sensation; PPD = purifed prote in deri wative (tuberculin test); PVD = peripheral vascular disease; RA = theumatoid arthrit $s ; \mathrm{RP}=$ retroperitoneal approach; $\mathrm{SBE}=$ subacute bacterial endocarditis; SLR = strajght eg raise positi 'e; TA = transabdominal approach; TP = transpedicular approach; UTI = urinary' tract infection; $X R T=$ radiation therapy.

t Cases of a second site of discitis requirino suraical inter wention because of unresconsiveness to ancrocriate antiti- 
All 20 cases were treated with vertebral arthrodesis: 12 cases with both interbody and posterolateral onlay arthrodesis, two with posterolateral onlay arthrodesis only, and six with interbody arthrodesis only. In the 18 interbody arthrodeses, we used the following bone grafts: two vascularized rib grafts, eight autologous bone (four rib, four tricortical iliac crest) and eight homoplastic bone (six fibula, two humerus). We used autologous bone (three rib, 11 iliac crest) in all 14 of the posterolateral onlay arthrodeses. The type of arthrodesis performed was individualized in each case. In general, cervical cases were treated with vertebral corpectomy and interbody arthrodesis, and thoracic and lumbar cases were treated with both interbody and posterolateral onlay arthrodeses. The two cases treated with posterolateral onlay arthrodesis alone were cases in which a posterior decompression was performed for primary discitis. The type of bone graft chosen was also individualized, based on the severity of the infection, the immune status of the patient, and the role of the bone graft in the vertebral construct.

Fifteen of the 20 cases of vertebral arthrodesis were augmented with spinal instrumentation. Caspar anterior cerebral plates with bicortical screw fixation were used in four of the six cervical cases. The two patients with noninstrumented cervical reconstruction were placed in a Halo orthosis. These two procedures were performed before we had gained confidence in placing instrumentation into infected cervical beds. Posterior segmental fixation was used in 11 of the 14 thoracic and lumbar cases. In four thoracic and one lumbar case, Texas Scottish Rite Hospital (TSRH) hook/rod constructs were used. Constructs extended two levels above and two levels below the involved spinal segment. When an interbody graft was used, the construct compressed across the graft and distracted over the remaining construct. In two thoracolumbar and four lumbar cases, TSRH transpedicle screw/rod constructs were used. If an interbody graft was used, a short segment construct extending one level above and one level below was placed. If no interbody graft was used the construct was extended to two levels above and two levels below the involved spinal segment. In general, hook/rod constructs were used in the thoracic spine, and pedicle screw/rod constructs were used in the lumbar spine. Five of the lumbar cases were treated in stages. Stage one involved an anterior approach (four retroperitoneal and one transabdominal) and vertebral corpectomy with interbody grafting. Stage two was performed 3 to 5 days later and involved a posterior approach with decompressive laminectomy (if necessary), posterolateral onlay bone grafting, and posterior segmental fixation. Whenever possible, the lateral extracavitary approach was used to accomplish both anterior and posterior decompression, as well as reconstruction, in one stage. 


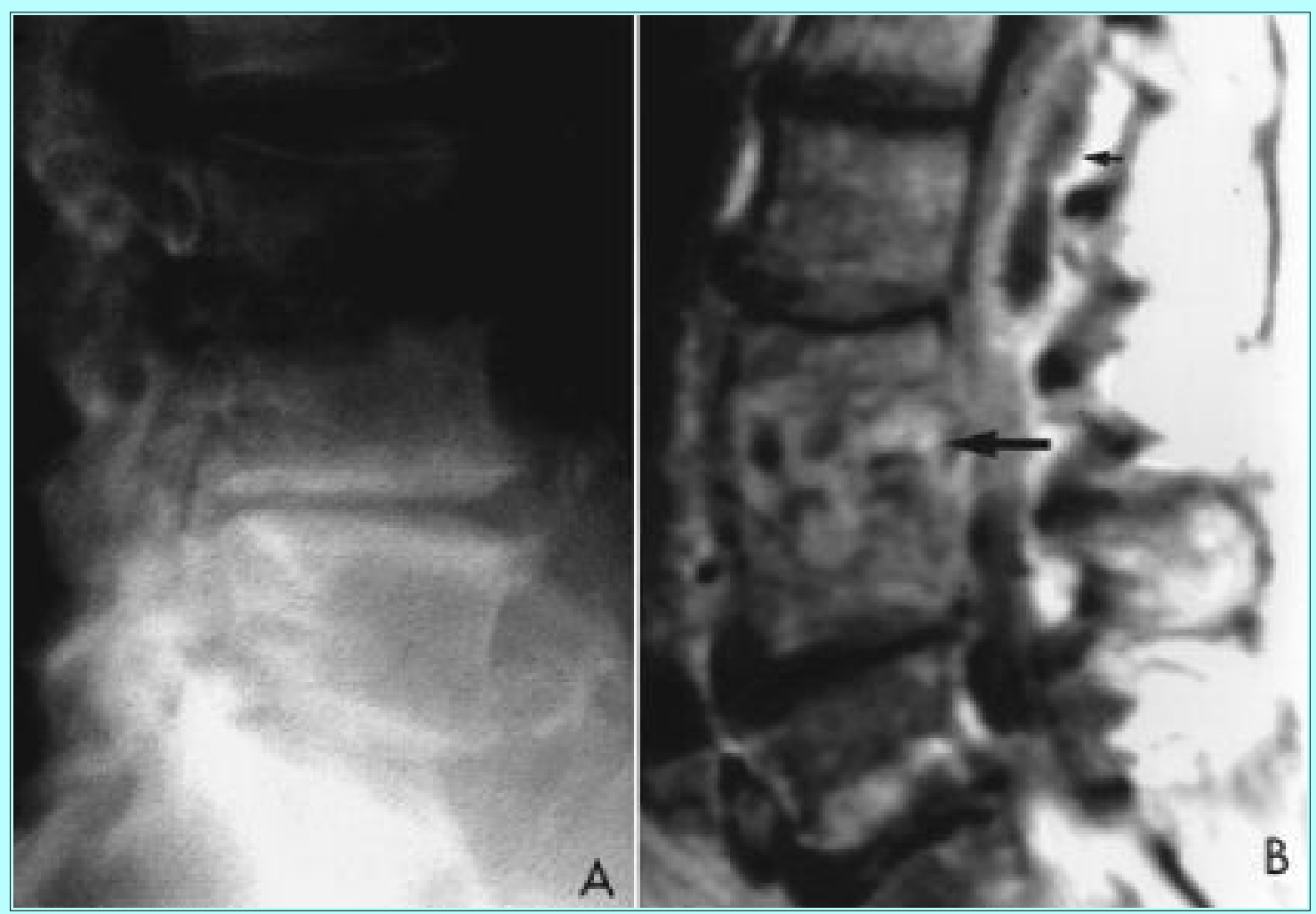

Fig. 1. Case 12. A: Preoperative plain lateral radiograph of the lumbar spine showing obliteration of the L3-4 disc space and erosion of the L-4 endplate and half of the L-3 vertebral body with loss of the lumbar lordosis. B: Preoperative sagittal gadolinium-enhanced $\mathrm{T}_{1}$-weighted $\mathrm{MR}$ image showing irregular enhancement in the area of the L3-4 disc space with dorsal protrusion into the ventral epidural space (large arrow). There is a noncontiguous dorsal epidural abscess rostral to the L3-4 disc space (small arrow). Also, note enhancement of the inferior endplate of L-5.

\section{ILLUSTRATIVE CASES}

\section{Case 12}

This 74-year-old man with insulin-dependent diabetes mellitus developed progressive paraparesis with neurogenic bladder. Examination showed focal midline back pain in the L3-4 interspinous area, decreased range of motion of lumbar spine, and marked back pain on straight leg raise maneuvers. Neurological examination showed a 3/5 paraparesis with nondermatomal lower-extremity sensory deficit and diminished cremasteric and anal sphincter reflexes and rectal muscular tone. The patient's preoperative erythrocyte sedimentation rate was $120 \mathrm{~mm} /$ hour. Radiological studies showed L3-4 disc space destruction with adjacent vertebral body destruction and loss of lumbar lordosis with mild left-sided spondylolisthesis (Fig. 1A). Magnetic resonance (MR) imaging showed osteomyelitic changes in the L-3 and L-4 vertebrae with associated ventral epidural and noncontiguous dorsal epidural abscesses (Fig. 1B). Emergency left-sided retroperitoneal L3-4 discectomy and complete L-3 and partial L-4 corpectomy were performed. An arthrodesis was performed using a humerus allograft. An associated iliopsoas abscess was drained directly via this approach. Intraoperative staining demonstrated Gram-positive cocci, which were identified in culture as Staphylococcus aureus. A course of intravenously administered nafcillin, $1 \mathrm{~g}$ every 8 hours, was initiated. Postoperative x-ray films obtained after mobilization in a clamshell orthosis showed displacement of the interbody graft (Fig. 2). 


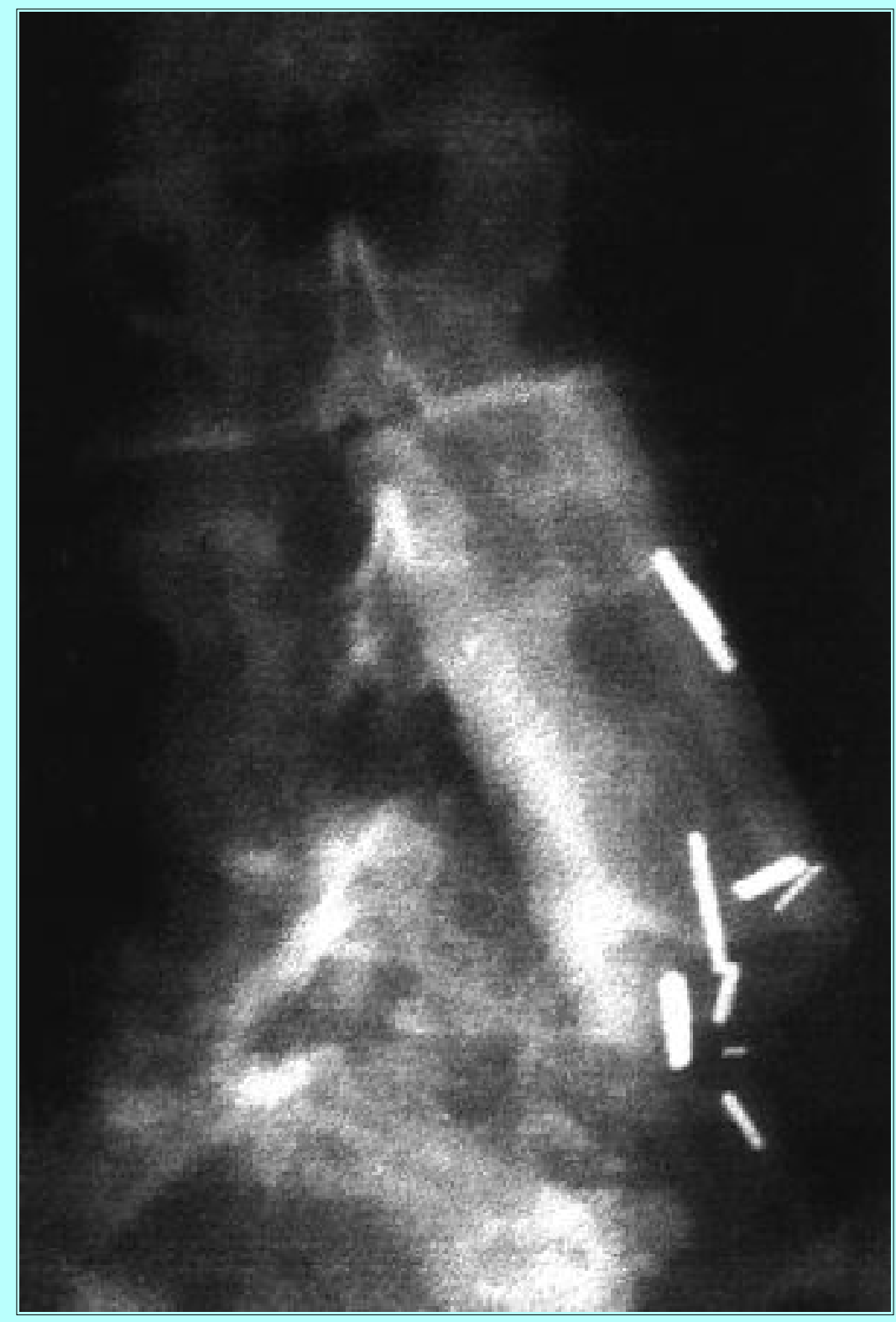

Fig. 2. Case 12. Plain anteroposterior lumbar radiograph obtained postoperatively showing displacement of the inferior aspect of the interbody humerus allograft. This displacement was identified on routine radiographs obtained after initial mobilization and was asymptomatic. For biomechanical reasons, the patient was returned to surgery ; a complete L-4 corpectomy was performed and a new humerus allograft was positioned from L2-5. A simultaneous posterior decompression, arthrodesis, and segmental fixation were performed as well.

Although the patient experienced some pain relief and had a stable neurological examination, we elected to reposition the interbody graft for biomechanical support and improved surface contact for arthrodesis. Using the same retroperitoneal exposure, we performed a complete L-4 corpectomy and positioned a new humerus interbody allograft. We performed the stage two posterior procedure, including L-3 and L-4 laminectomy drainage of the dorsal epidural abscess and placement of posterolateral autologous iliac crest bone graft from L2-5, before additional anesthesia became necessary. We also performed L2-5 posterior segmental fixation using a TSRH pedicle screw/rod construct (Fig. 3). 


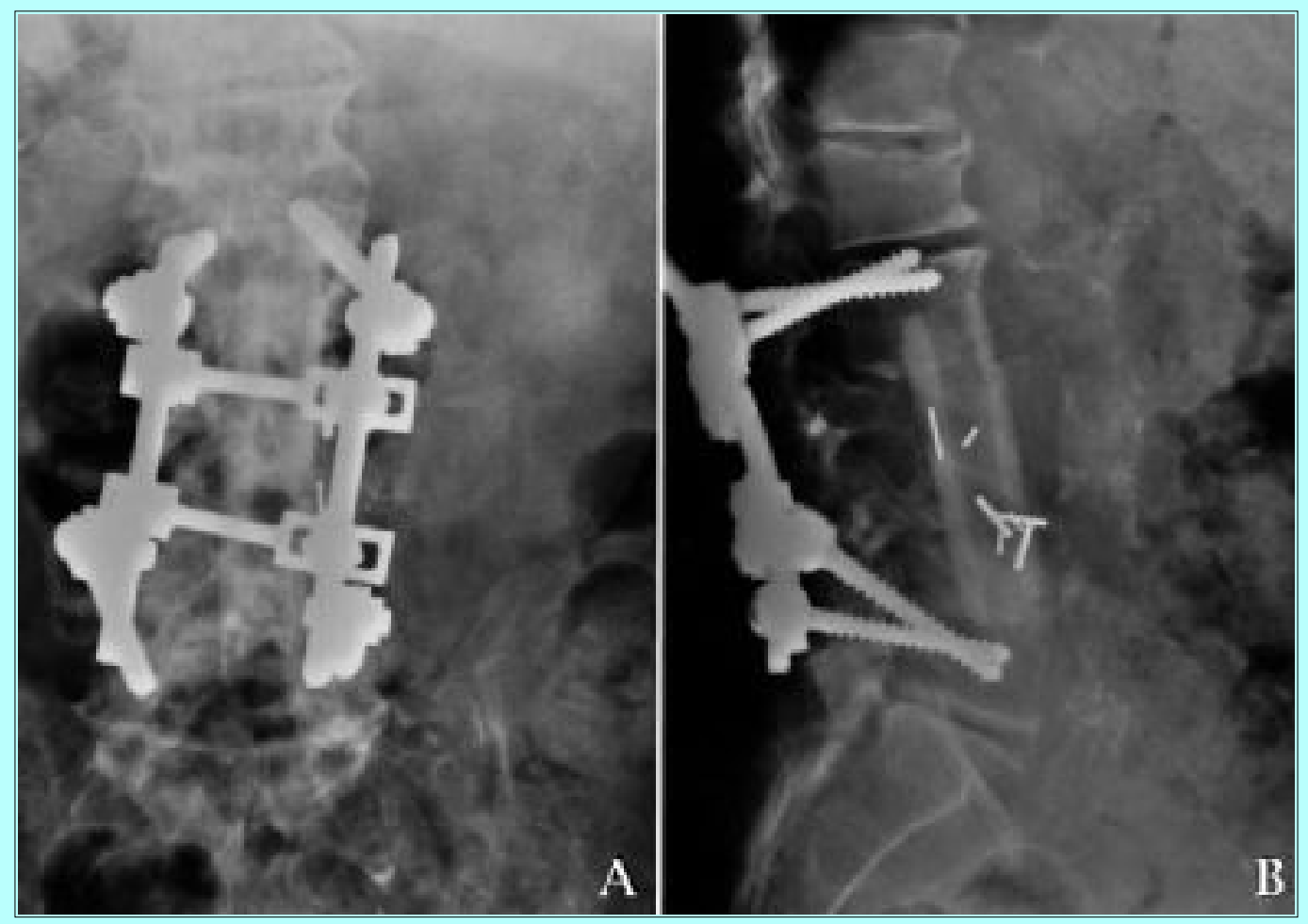

Fig. 3. Case 12. Anteroposterior (A) and lateral (B) lumbar plain radiographs obtained 25 months postoperatively, showing good arthrodesis. The interbody graft has telescoped somewhat but is fused well at the endplates and the posterior segmental instrumentation is intact. Despite developing a new infection with $S$. aureus in the hip, the patient did not develop recurrent spinal infection. The neurological examination was normal.

For 3 months postoperatively the patient used a clamshell orthosis when the spine was weight-bearing. During the first 6 weeks his back pain resolved, strength improved to 4/5, and bladder control returned. He was treated with a 3-month course of parenterally administered nafcillin followed by a 3-month course of oral dicloxacillin (500 mg every 6 hours). His sedimentation rate returned to normal in 3 months, and at 6 months radiographic studies demonstrated stable arthrodesis. His neurological examination was normal at 25 months.
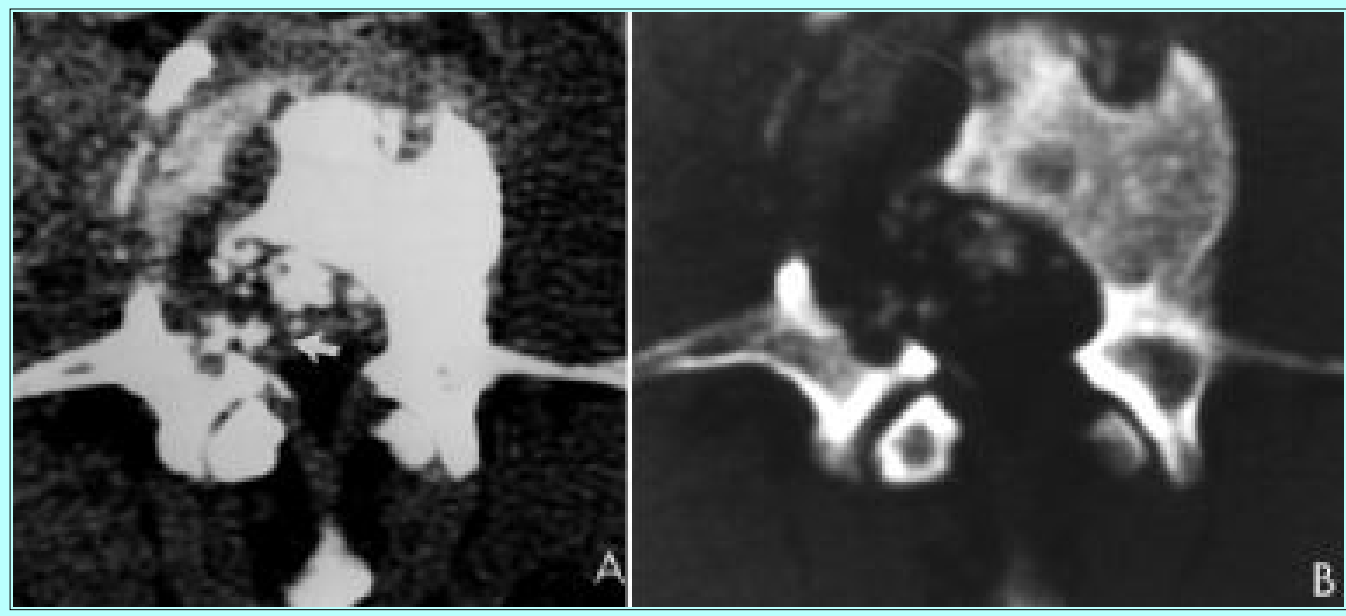

Fig. 4. Case 10. Preoperative axial CT soft tissue (A) and bone window (B) images of the L-2 body at the pedicle level showing destruction of the right side of the vertebral body and pedicle. A soft tissue mass extends from the destroyed vertebral body into the right ventrolateral epidural space with deflection of the thecal sac to the left and posterior (arrow). 
There was extensive L1-2 discitis with osteomyelitis and prevertebral, epidural, and iliopsoas abscesses.

\section{Case 10/11}

This 57-year-old woman with steroid-dependent rheumatoid arthritis was admitted to the hospital for sepsis secondary to pyelonephritis. Her blood cultures were positive for $S$. aureus and a course of parenteral antibiotic drugs was initiated. Over the next week she developed progressive low-back pain and severe radicular pain in her left upper thigh. A physical examination revealed diffuse marked lumbar back pain and bilateral leg pain with straight leg raising test. Neurological examination revealed $4 / 5$ strength in the anterior tibialis and extensor hallucis longus bilaterally and absent achilles reflexes and bilateral extensor Babinski reflexes. Radiological studies showed destructive changes based at the endplates adjacent to the L1-2 disc and increased endplate sclerosis with loss of disc space height at the L4-5 disc (Fig. 4). Bilateral iliopsoas muscle abscesses were drained percutaneously with the computerized tomography (CT)-guided technique. Cultures were positive for $S$. aureus and a course of intravenously administered vancomycin ( $1 \mathrm{~g}$ every 12 hours) was initiated. After the patient stabilized medically, she underwent staged surgical procedures. First, L-1 and L-2 corpectomies and a humerus allograft interbody arthrodesis were performed via a retroperitoneal approach. Five days later, a posterior T12-L3 onlay arthrodesis using autologous iliac crest bone grafts and T12-L3 segmental fixation using a TSRH pedicle screw/rod construct were performed (Fig. 5). For 3 months postoperatively, she used a clamshell orthosis when the spine was weight-bearing. The patient regained full motor strength and her achilles reflexes returned during a 6-week interval. She was treated with intravenously administered antibiotic drugs ( $1 \mathrm{~g}$ every 12 hours) for 3 months and then received oral dicloxacillin (500 mg every 6 hours).

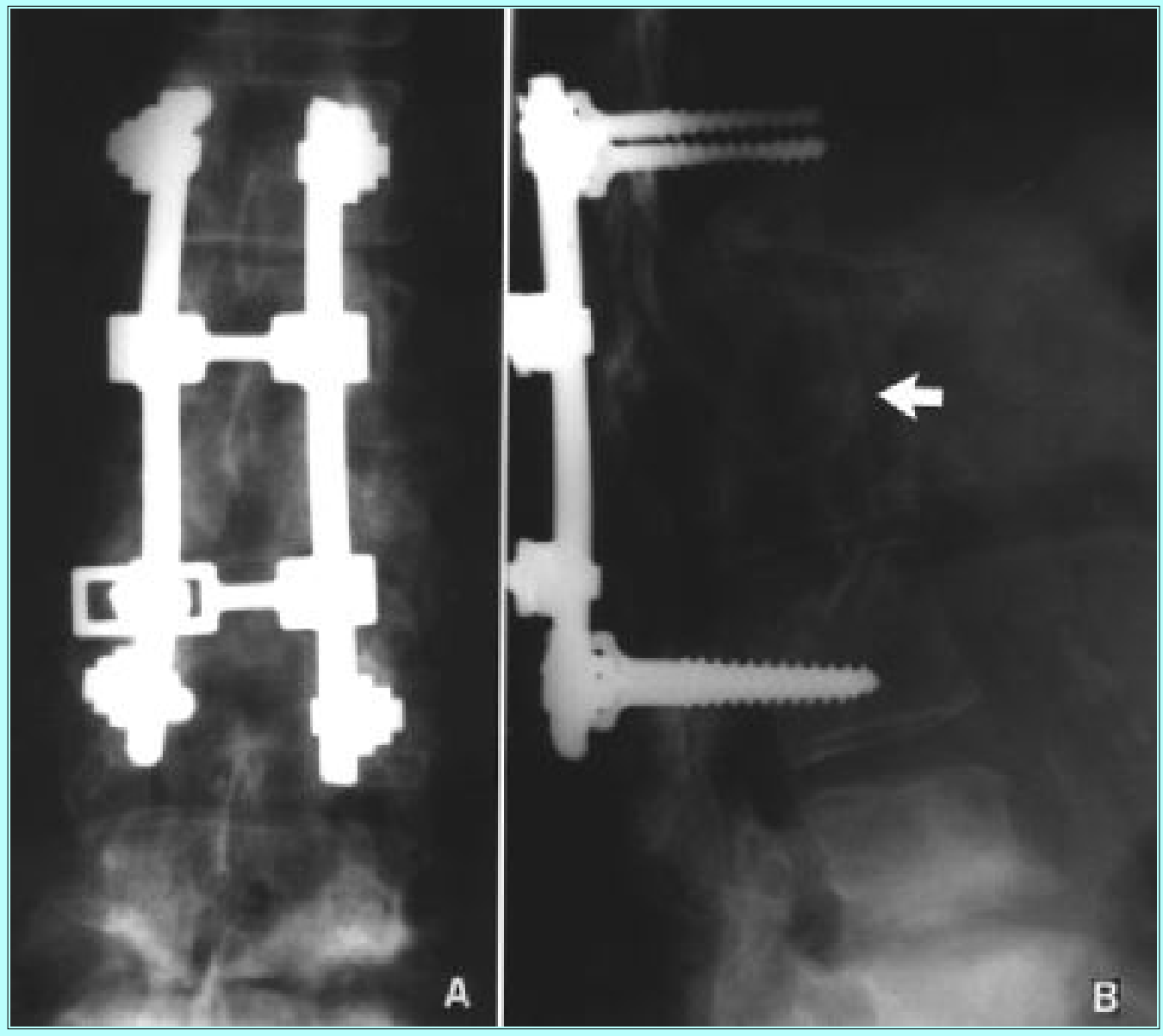


Fig. 5. Case 10. Postoperative anteroposterior (A) and lateral (B) lumbar plain radiographs obtained 6 weeks postoperatively showing intact posterior segmental instrumentation with pedicle screws in T-12 and L-3. The outline of the humerus interbody allograft can be seen extending from the T-12 to the L-3 vertebra (arrow). Note the advanced sclerosis and endplate irregularities with loss of disc space height at the L4-5 disc space. The L4-5 discitis showed progression on radiographic studies, although the clinical situation had returned to normal and the sedimentation rate was normal, indicating that radiographic changes can lag behind the clinical outcome.

After treatment for 1 month with oral antibiotic drugs (4 months postoperatively) she experienced increasing low-back pain. A physical examination demonstrated new low-back pain and loss of her returned achilles reflexes; her motor strength was normal. Radiological studies demonstrated progressive endplate sclerosis and loss of height at the L4-5 disc space along with a persistent central disc bulge accompanied by granulation tissue. There was another iliopsoas abscess on the left side. The sedimentation rate, which had returned to normal, had again increased to $70 \mathrm{~mm} /$ hour. Another set of staged surgical procedures was performed. First, an L4-5 discectomy and partial L-4 corpectomy were performed via a retroperitoneal approach with evacuation of the iliopsoas abscess and reconstruction with autologous tricortical iliac crest interbody arthrodesis. Next, a posterior L3-S1 onlay arthrodesis was performed using an autologous iliac crest bone graft, and L3-S1 segmental fixation was attached to the existing construct. For 3 months postoperatively the patient used a clamshell orthosis when the spine was weight-bearing. After 6 weeks she was found to have resolution of her back pain and normal motor strength. She was treated with another 3-month course of parenterally administered vancomycin followed by 3 months of oral dicloxacillin ( $500 \mathrm{mg}$ every 6 hours). The sedimentation rate returned to normal after 3 months. At 3 months there was radiographic evidence of loosening of one of the sacral screws, but by 6 months this screw was stable. Twenty-six and 34 months after her last and first operations, respectively, this patient died of metastatic cancer. The medical work up of her cancer did not show evidence of recurrent spinal infection (Fig. 6). 


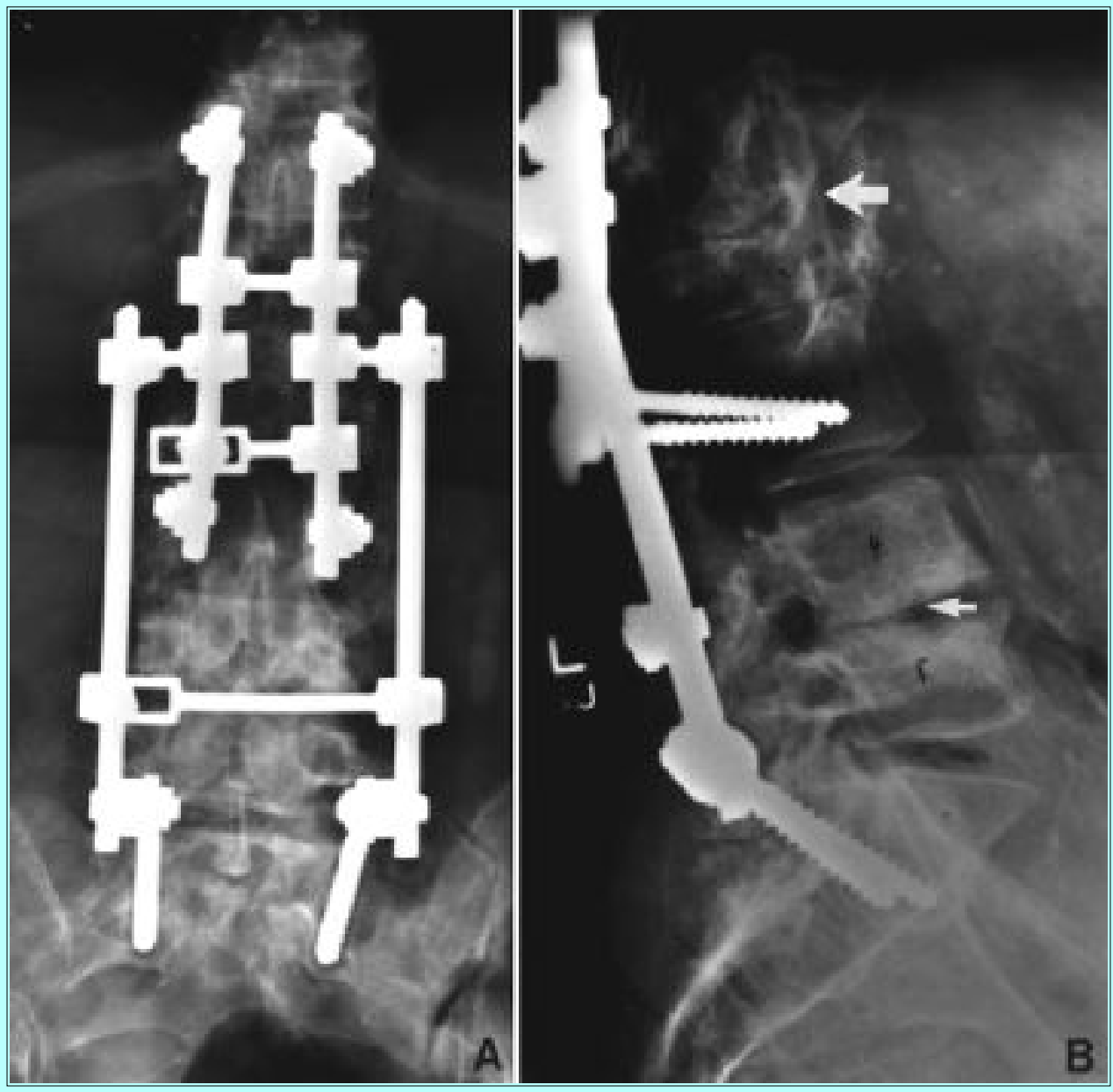

Fig. 6. Case 10/11. A and B: Anteroposterior and lateral lumbar plain radiographs obtained 24 months postoperatively showing the prior T12-L3 interbody humerus allograft with evidence of incorporation (large arrow) and intact T12-L3 posterior segmental instrumentation, and the new posterior segmental instrumentation with sacral pedicle screw fixation and linkage to the prior construct to create a conjoined construct fixating T12-S1. The L4-5 iliac crest interbody autologous graft cannot be visualized well (small arrow).

\section{RESULTS}

\section{Epidemiological Findings}

The mean age of the patients in this series was 54 years, with a range of 35 to 76 years. There was an equal male/female ratio. The main risk factors for spinal infections in this series were: immunocompromise (caused by steroids, cancer, chronic renal failure, positive purified protein derivative) in eight patients, urinary tract infections in seven (23\%), diabetes mellitus in five, obesity in five, and smoking in five. The presenting symptoms were dominated by pain (primarily axial) and motor deficits. However, myelopathic signs were present in 11 of 15 cases with infection between C-1 and L-2. The sedimentation rate was elevated in all patients except one, with an average value of $92 \mathrm{~mm} / \mathrm{hour}$. Leukocytosis with a granulocytosis was present in $50 \%$ of cases. Blood cultures were positive in four cases $(20 \%)$, and S. aureus was the primary pathogen in this series, occurring in $12(60 \%)$ of 20 cases. There were two cases of tuberculosis infections (10\%), two infections yielding bacteria containing Gram-negative rods (Salmonella and Proteus species) (10\%), one with S. epidermidis (5\%), and three negative cultures (15\%). There was a fairly equal distribution of spinal column involvement: six cerebral, eight thoracic, and six lumbar cases. 


\section{Radiological Findings}

Plain radiographs showed various conditions, from loss of cervical lordosis with prevertebral swelling to frank vertebral destruction with collapse, spondylolisthesis, and angulation. The most prominent and consistent finding was endplate erosion. Magnetic resonance imaging with and without gadolinium enhancement was the diagnostic study of choice and verified the inflammatory focus evolving from the disc space, the presence of prevertebral, ventral and dorsal epidural abscesses, and in some cases revealed the presence of iliopsoas abscesses. Contrast-enhanced CT scanning was used to evaluate posterior mediastinal and retroperitoneal abscesses fully and to guide percutaneous drainage of these abscesses. Discitis with endplate erosion was the primary finding in nine of the 20 cases, and discitis with osteomyelitis was the primary finding in the remaining 11 cases. An associated epidural abscess (gadolinium-enhanced fluid collection) was identified in nine (45\%) of 20 cases, and associated iliopsoas abscesses were identified in three (15\%) of 20 cases. All of the epidural abscesses were ventral and contiguous with the discitis except for one dorsal abscess that was present one level above the discitis and osteomyelitis.

\section{Clinical Findings}

Axial pain improved or resolved in all patients. In two of seven patients, radicular burning dysesthesia did not improve. Motor deficits were rated according to the Frankel motor classification,[12] as follows: Grade A, complete motor and sensory loss; Grade B, complete motor and incomplete sensory loss; Grade $\mathrm{C}$, some motor and incomplete sensory loss; Grade D, useful motor function and incomplete sensory loss; and Grade E, normal motor and sensory function. Preoperatively, two patients were classified as Frankel Grade A, one as Grade B, six as Grade C, seven as Grade D, and four as Grade E. Postoperatively, there was one patient in Frankel Grade A, none in Grade B, one in Grade C, five in Grade D, and 13 in Grade E. No patient was made worse by surgery. Six (30\%) of 20 cases (four of which were Grade E) had an unchanged Frankel grade postoperatively (Table 2). Seven (35\%) of 20 cases improved one grade, six (30\%) of 20 improved two grades, and one (5\%) of 20 cases improved three grades postoperatively. Myelopathy improved in all cases except for one patient with a complete spinal cord injury. In five of seven patients with neurogenic bladders, the condition resolved postoperatively. 
TABLE 2

FRANKEL CLASSF CATION FOR 20 CASES OF SPINAL INFECTION TREATED WITH ARTHRODESB*

\begin{tabular}{|c|c|c|c|c|c|}
\hline $\begin{array}{l}\text { Case } \\
\text { No. }\end{array}$ & Preop Exam & Grade & Pcotop Exam & Grade & Change \\
\hline $\begin{array}{l}1 \\
2 \\
3\end{array}$ & $\begin{array}{l}45 \text { hand pare sis, propriocepti ue loss } \\
35 \text { IP paresis, T-9PP loss } \\
35 \text { paraparesis, T6-7 } \\
\text { PP loss, proprioceptive loss }\end{array}$ & $\begin{array}{l}\mathrm{D} \\
\mathrm{C} \\
\mathrm{C}\end{array}$ & $\begin{array}{l}\text { hand \& propriocepoption nomal } \\
\text { IP \& sensation normal } \\
\text { motor \& PP normal, } \\
\text { proprioceptive loss }\end{array}$ & $\begin{array}{l}\mathrm{E} \\
\mathrm{E} \\
\mathrm{E}\end{array}$ & $\begin{array}{c}1+ \\
2+ \\
2+\end{array}$ \\
\hline 4 & 25 paraparesis & $\mathrm{C}$ & motor norm al & $E$ & $2+$ \\
\hline 5 & neuro norm al & $\mathrm{E}$ & neuro norm al & $\mathrm{E}$ & 0 \\
\hline 6 & L-2 paraplegia & A & died & A & 0 \\
\hline 7 & 35 paraparesis, T-6 PP loss & $\mathrm{C}$ & motor norm al, T-6 PP im pro ued & $\mathrm{E}$ & $2+$ \\
\hline 8 & 45 paraparesis & $\mathrm{D}$ & motor normal & $E$ & $1+$ \\
\hline 9 & 35 monoparesis & $\mathrm{D}$ & motor norm al & $\bar{E}$ & $1+$ \\
\hline 10 & 45 foot drop & $\mathrm{D}$ & motor normal & $E$ & $1+$ \\
\hline 11 & neuro norm al & $\vec{E}$ & neuro norm al & $\mathrm{E}$ & 0 \\
\hline 12 & 35 paraparesis & $\mathrm{C}$ & motor normal & $\mathrm{E}$ & $2+$ \\
\hline 13 & neuro norm al & $E$ & neuro norm al & $\bar{E}$ & 0 \\
\hline 14 & 25 IP paresis, 45 paraparesis & $\mathrm{C}$ & 35 IP paresis & $\mathrm{D}$ & $1+$ \\
\hline 15 & arm monoplegia. & $\mathrm{C}$ & 45 monoparesis & $\mathrm{D}$ & $1+$ \\
\hline 16 & -4 quadraplegia & A & C-6 quadraplegia & $\mathrm{C}$ & $2+$ \\
\hline 17 & neuro norm al & $\mathrm{E}$ & neuro norm al & $\mathrm{E}$ & 0 \\
\hline 18 & 35 arm monoparesis, paraplegia & $\mathrm{B}$ & neuro norm al & $\mathrm{E}$ & $3+$ \\
\hline 19 & 45 paraparesis, L-1 PP loss & $\mathrm{D}$ & 45 paraparesis, PP normal & $\mathrm{D}$ & 0 \\
\hline 20 & 45 arm monoparesis & $\mathrm{D}$ & neuro norm al & $\mathrm{E}$ & $1+$ \\
\hline
\end{tabular}

\section{Surgical and Arthrodesis Complications}

The average radiographic follow-up time was 16 months for 17 of the 20 cases. Two patients with interbody and onlay arthrodeses were lost to follow up and were not included. One patient with interbody and onlay arthrodesis died in the postoperative period and was also not included. For the 17 of 20 patients with more than 6 months of radiographic follow-up review, all nine with autologous interbody grafts showed evidence of bone fusion. Five of six homoplastic interbody grafts showed evidence of bone fusion. In one cervical case mild telescoping of the fibular graft and development of an asymptomatic pseudarthrosis were seen. All 11 autologous onlay grafts showed evidence of bone fusion. There were no cases of allograft onlay fusion.

Surgical complications occurred in four (20\%) of 20 cases. Three of these complications were considered minor: one superficial wound infection, one superficial partial dehiscence, and one corneal abrasion. There was one deep wound infection that required surgical debridement (this same patient later developed the superficial wound dehiscence). Complications of the arthrodesis were identified on radiographic studies in four $(20 \%)$ of 20 cases. One cerebral interbody fibular allograft telescoped and developed an asymptomatic pseudarthrosis. One sacral pedicle screw seemed loose but was asymptomatic and the graft displayed solid bone fusion. One asymptomatic partially dislodged lumbar interbody humerus allograft was repositioned surgically. Finally, one patient developed a delayed, progressive thoracic kyphosis that caused new axial pain. This patient decided to forego surgical intervention.

\section{Medical Complications}

Medical complications occurred in five (25\%) of 20 cases: one congestive heart failure, one pneumonia, one atrial fibrillation, and two urinary tract infections. These combined surgical, arthrodesis, and medical 
complications occurred in $10(50 \%)$ of 20 cases. However, only two (10\%) of these complications required reoperation.

There was one death in the early postoperative period, in a 50-year-old man with coronary artery disease, chronic atrial fibrillation, subacute bacterial endocarditis, congestive heart failure, morbid obesity, and severe peripheral vascular disease. Medical consultants predicted a 50\% chance of death if surgery was performed. However, he requested surgical treatment for paraplegia that persisted despite maximum medical therapy. Ten days postoperatively, he developed Gram-negative sepsis from an extraspinal source, liver failure, and bilateral lower-extremity gangrene. Despite aggressive critical care he died of multiple organ failure.

\section{Recurrence Rate}

The duration of clinical follow up was greater than 2 years in $15(75 \%)$ of 20 cases, with an average follow-up period postsurgery of 37 months. Fourteen of 15 patients were free of infection after discontinuation of antibiotic drugs, at an average of 31 months (Table 3). One patient developed an infected hip joint 7 months after discontinuation of antibiotic drugs and was treated with another prolonged course of antibiotic medication. No recurrent spinal infections were documented.

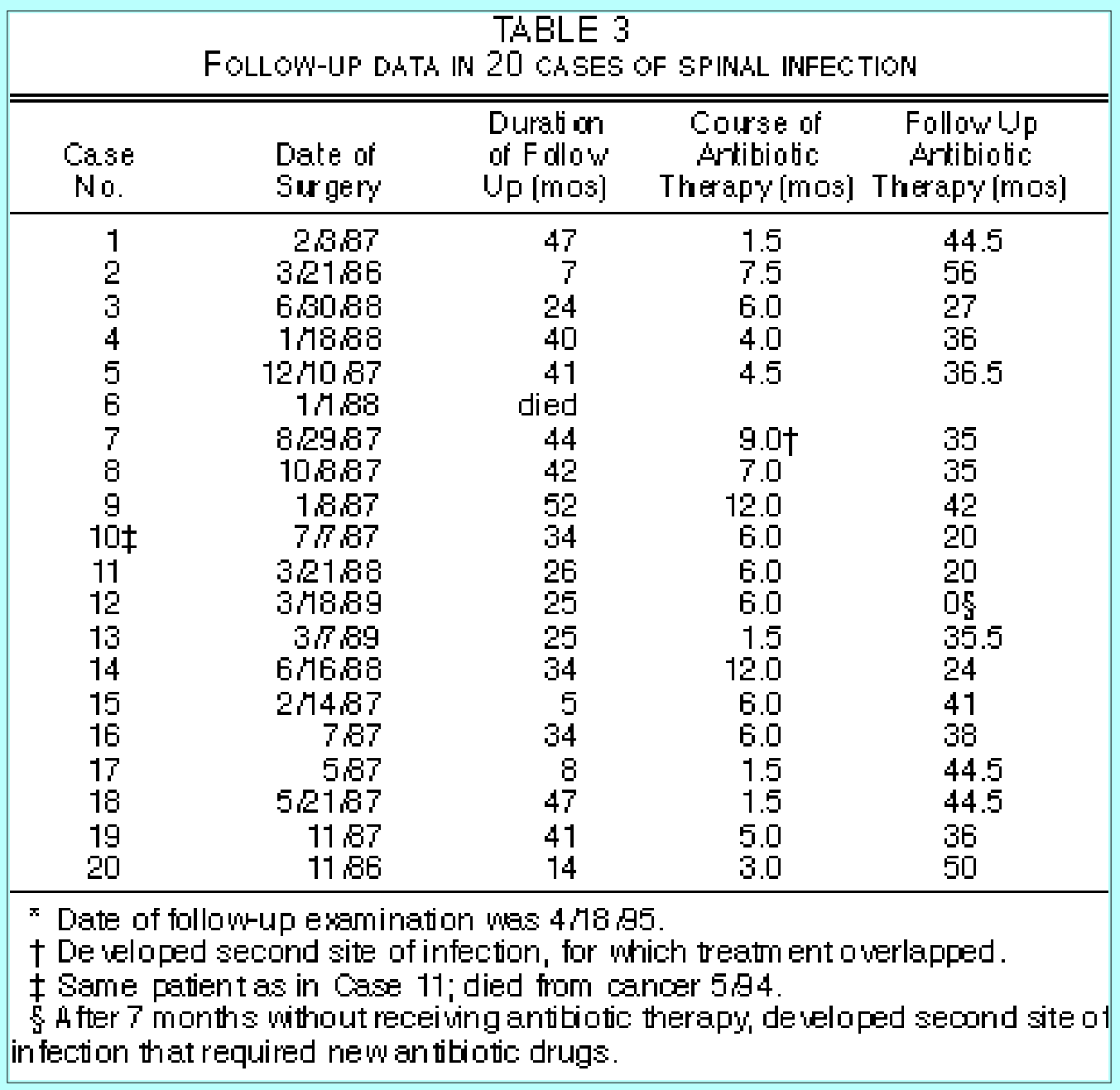

\section{Special Note}

There were three cases of multiple spinal foci (15\%). In all three cases the second spinal focus did not respond to appropriate medical therapy. Two of these patients required spinal reconstruction and are 
presented in this series. There were no cases of a new secondary focus developing after treatment of the primary focus.

\section{DISCUSSION}

This surgical series is a biased selection of cases representing advanced infections of the spine with progressive clinical presentations. However, this series is comparable to prior medical series in the following areas: 1) the average age was older than 50 years;[30] 2) immunocompromised and diabetic patients seem particularly susceptible to infections;[14,30] 3) urinary tract infection is a frequent source;[15,35] 4) $S$. aureus is the primary pathogen; 5) multiple pathogens are rare;[13,30] and 6) the predominant presenting complaint is pain.[30] This series differs from prior medical series in that there is not a preponderance of males or of lumbar spine involvement; [30] abscess formation is not increased in the cervical or thoracic spine;[14] there is a high incidence of neurological deficits; $[9,30]$ and significant spinal deformity is a common finding.[13,21]

The management of these infections was aggressive. It included complete surgical debridement of infected foci, spinal reconstruction with arthrodesis and instrumentation, and prolonged antibiotic therapy. Antibiotic therapy involved 6 to 12 weeks of parenterally administered antibiotic drugs and 3 months of oral antibiotic medications that were selected based on identification of pathogens and their sensitivities in culture.[13,23,30]

An anterior surgical approach was used to allow direct access to the infectious foci for aggressive debridement.[9,17-20] Aggressive anterior debridement, however, requires reconstruction to prevent spinal deformity. Autologous interbody bone grafting in the presence of active infection was first reported by Wiltberger[38] in 1952 for chronic vertebral osteomyelitis and has been used safely since then.[10,17,18,20] Although autologous iliac crest bone grafts are preferred,[17,18,28] vascularized rib grafts have been used successfully and fibula grafts have been shown to be effective in the cervical spine. $[1,37]$ Posterior stabilization and fusion are indicated for significant spinal deformities or for procedures performed at the thoracolumbar junction.[11,18,19,22,28]

The use of spinal instrumentation in the context of infection has not been thoroughly addressed in the literature. Currier and Eismont[5] reported instrumentation of the thoracolumbar spine for T10-11 discitis and osteomyelitis with associated epidural abscess, but did not discuss the role of spinal instrumentation in this context. It has been reported previously that instrumentation should not be used in the presence of active infection, but a clear explanation for this statement was not provided.[3] The most extensive report of the use of spinal instrumentation of the infected spine presented six cases (two tubercular and four pyogenic) of advanced spinal infections.[29] These cases were treated with drainage of the infected foci and stabilization with a Banks-Dervin rod.[26] One patient had superficial wound infection and two patients died (one at 6 months of cardiac failure, one at 25 days of unresponsive pneumonia). Three patients received follow-up care for 12 months and one for 30 months (tuberculosis treated with chemotherapy for 24 months). There was no evidence of recurrent infections.

Clearly, the use of spinal instrumentation increases the potential for the development of postoperative infection.[25,27,33] Pathobiological studies indicate multiple variables responsible for induction of infection: foreign body-associated tissue damage, impairment of host defenses, bacterial trapping by fibrin, sequestration of bacteria in implant interstices, and the generation of a biofilm on implant surfaces. $[2,8,16]$ It is not entirely clear whether this information contraindicates the use of spinal instrumentation in the presence of infection. Intuitively, if the implant is exposed to bacteria, its surface 
must become impregnated and increase the risk of developing an infection. However, there is clinical evidence indicating that this may not be absolutely inevitable. Two reports detail the successful treatment of infected spinal instrumentation without its removal.[7,32] Furthermore, the cases of multiple infectious foci presented in this series are revealing in this regard. These are cases in which active secondary foci failed to respond to appropriate medical management, but did not cause infection of the spinal instrumentation or recurrent infection at the original site.

The duration of follow-up study necessary to demonstrate successful eradication of osteomyelitis is not well established. Delayed recurrence has been reported more than 1 year postsurgery.[4,10] In their study of adult osteomyelitis, Cierny, et al.,[4] reported that indium scans are positive in $87 \%$ of patients at 1 year and in $6 \%$ at 2 years. Nevertheless, they found only two treatment failures beyond 1 year. The time after antibiotic medication is discontinued in relation to this follow-up period also has not been clearly addressed. The present study has a follow-up period of 37 months postsurgery and 31 months after antibiotic medication was discontinued, representing $75 \%$ of the cases.

\section{CONCLUSIONS}

Vertebral reconstruction can be performed safely as part of the primary surgical procedure. The preferred graft for arthrodesis of the infected spine is an autologous graft, but allografts are not contraindicated. Spinal instrumentation can be placed safely as part of the primary procedure and should not be considered contraindicated. However, the indications for spinal instrumentation should be very specific and placement should be reserved for clear spinal instability. Whether spinal instrumentation should be removed after bone arthrodesis is not clearly answered by this study. The possibility of very delayed symptomatic cryptic infection of the instrumentation may still exist.[31]

\section{References}

1. Bradford DS, Daher YH: Vascularised rib grafts for stabilisation of kyphosis. J Bone Joint Surg Br 68:357-361, 1986

2. Buret A, Ward KH, Olson ME, et al: An in vivo model to study the pathobiology of infectious biofilms on biomaterial surfaces. J Biomed Mater Res 25:865-874, 1991

3. Camins MB, Cooper PR: Spinal Infections. Neurosurgical Consultations 4, 1993

4. Cierny G, Mader JT, Penninck JJ: A clinical staging system for adult osteomyelitis. Contemp Orthop 10:17-37, 1985

5. Currier BL, Eismont FJ: The Spine. 1992, pp 1319-1380

6. Denis F: The three column spine and its significance in the classification of acute thoracolumbar spinal injuries. Spine 8:817-831, 1983

7. Dietze DD Jr, Haid RW Jr: Antibiotic-impregnated methylmethacrylate in treatment of infections with spinal instrumentation: case report and technical note. Spine 17:981-987, 1992

8. Dougherty SH: Pathobiology of infection in prosthetic devices. Rev Infect Dis 10:1102-1117, 1988

9. Eismont JF, Bohlman HH, Soni PL, et al: Pyogenic and fungal vertebral osteomyelitis with paralysis. J Bone Joint Surg Am 65:19-29, 1983 
10. Emery SE, Chan DPK, Woodward HR: Treatment of hematogenous pyogenic vertebral osteomyelitis with anterior debridement and primary bone grafting. Spine 14:284-291, 1989

11. Fountain SS: A single-stage combined surgical approach for vertebral resections. J Bone Joint Surg (Am) 61:1011-1017, 1979

12. Frankel HL, Hancock DO, Hyslop G, et al: The value of postural reduction in the initial management of closed injuries of the spine with paraplegia and tetraplegia. Paraplegia 7:179-192, 1969

13. Frederickson B, Yuan H, Orlans R: Management and outcome of pyogenic vertebral osteomyelitis. Clin Orthop 131:160-167, 1978

14. Garcia A Jr, Grantham SA: Hematogenous pyogenic vertebral osteomyelitis. J Bone Joint Surg Am 42:429-436, 1960

15. Genster HG, Andersen MJF: Spinal osteomyelitis complicating urinary tract infection. J Urol 107:109-111, 1972

16. Gristina AG, Naylor P, Quentin M: Infections from biomaterials and implants: a race for the surface. Med Prog Technol 14:205-224, 1988

17. Hodgson AR, Stock FE: Anterior spine fusion for the treatment of tuberculosis of the spine: the operative findings and results of treatment in the first one hundred cases. J Bone Joint Surg Am 42:295-310, 1960

18. Kemp HBS, Jackson JW, Jeremiah JD, et al: Anterior fusion of the spine for infective lesions in adults. J Bone Joint Surg (Br) 55:715-734, 1973

19. Kemp HBS, Jackson JW, Shaw NC: Laminectomy in paraplegia due to infective spondylosis. Br J Surg 61:66-72, 1974

20. Kirkaldy-Willis WH, Thomas TG: Anterior approaches in the diagnosis and treatment of infections of the vertebral bodies. J Bone Joint Surg Am 47:87-110, 1965

21. Kulowski J: Pyogenic osteomyelitis of the spine. An analysis and discussion of 102 cases. J Bone Joint Surg 18:343-364, 1936

22. La Rocca H: Spinal sepsis, in Rothman RH, Simeone FA (eds): The Spine, ed 2. Philadelphia: WB Saunders, 1982, pp 757-774

23. Mader JT, Cantrell JS, Calhoun J: Oral ciprofloxacin compared with standard parenteral antibiotic therapy for chronic osteomyelitis in adults. J Bone Joint Surg Am 72:104-110, 1990

24. Malawski SK, Lukawski S: Pyogenic infections of the spine. Clin Orthop 272:58-66, 1991

25. Micheli LJ, Hall JE: Complications in the management of adult spinal deformities, in Epps CH Jr (ed): Complications in Orthopaedic Surgery, ed 2. Philadelphia: JB Lippincott, 1986, Vol 2, pp 1227-1229

26. Miles J, Banks AJ, Dervin E, et al: Stabilisation of the spine affected by malignancy. J Neurol Neurosurg Psychiatry 47:897-904, 1984 
27. Moe JH: Complications of scoliosis treatment. Clin Orthop 53:21-30, 1967

28. Rajasekaran S, Soundarapandian S: Progression of kyphosis in tuberculosis of the spine treated by anterior arthrodesis. J Bone Joint Surg Am 71:1314-1323, 1989

29. Redfern RM, Miles J, Banks AJ, et al: Stabilisation of the infected spine. J Neurol Neurosurg Psychiatry 51:803-807, 1988

30. Sapico FLM, Montgomerie JZM: Vertebral osteomyelitis. Infect Dis Clin North Am 4:539-550, 1990

31. Smith MM, Vasseur PB, Saunders HM: Bacterial growth associated with metallic implants in dogs. J Am Vet Med Assoc 195:765-767, 1989

32. Thalgott JS, Cotler HB, Sasso RC, et al: Postoperative infections in spinal implants: classification and analysis--a multicenter study. Spine 16:981-984, 1991

33. Transfeldt EE, Lonstein JE: Wound infections in elective reconstructive spinal surgery. Orthop Trans 9:128-129, 1985

34. Waldvogel FA, Medoff G, Swartz MN: Osteomyelitis: a review of clinical features, therapeutic considerations and unusual aspects. N Engl J Med 282:198-206, 260-266, 316-322, 1970

35. Wedge JH, Oryschak AF, Robertson DE, et al: Atypical manifestations of spinal infections. Clin Orthop 123:155-163, 1977

36. White AA III, Panjabi MM: Clinical Biomechanics of the Spine. Philadelphia: JB Lippincott, 1978

37. Whitecloud TS, LaRocca H: Fibular strut graft in reconstructive surgery of the cervical spine. Spine 1:33-43, 1976

38. Wiltberger BR: Resection of vertebral bodies and bone-grafting for chronic osteomyelitis of the spine. A case report. J Bone Joint Surg Am 34:215-218, 1952

Manuscript received May 3, 1996.

Accepted in final form December 10, 1996.

This series was presented to the spine section of the General Scientific Session at the Annual Meeting of the American Association of Neurological Surgeons in Boston, Massachusetts, April 27, 1993. The present updated series was presented to the Spine Section Session of the Annual Meeting of the Congress of Neurological Surgeons in San Francisco, California, October 18, 1995.

Address reprint requests to: Richard G. Fessler, M.D., Ph.D., Department of Neurosurgery, University of Florida, P.O. Box 100265, J. Hillis Miller Health Science Center, Gainesville, Florida 32610-0265. 